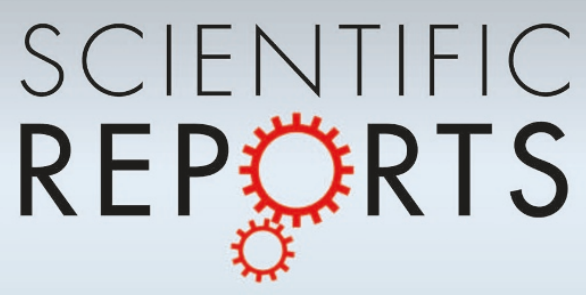

OPEN

SUBJECT AREAS:

SURFACES, INTERFACES

AND THIN FILMS

INFORMATION STORAGE

SPINTRONICS

MAGNETIC PROPERTIES AND MATERIALS

Received

25 March 2013

Accepted

27 August 2013

Published

25 September 2013

Correspondence and requests for materials should be addressed to N.A.P. (pertsev. domain@mail.ioffe.ru)

\section{Origin of easy magnetization switching in magnetic tunnel junctions with voltage-controlled interfacial anisotropy}

\author{
Nikolay A. Pertsev
}

\author{
A. F. loffe Physical-Technical Institute of the Russian Academy of Sciences, 194021 St. Petersburg, Russia.
}

Spin-polarized currents represent an efficient tool for manipulating ferromagnetic nanostructures but the critical current density necessary for the magnetization switching is usually too high for applications. Here we show theoretically that, in magnetic tunnel junctions having electric-field-dependent interfacial anisotropy, the critical density may reduce down to a very low level $\left(\sim 10^{4} \mathrm{~A} \mathrm{~cm}^{-2}\right)$ when the junction combines small conductance with the proximity of free layer to a size-driven spin reorientation transition. The theory explains easy magnetization switching recently discovered in $\mathrm{CoFeB} / \mathrm{MgO} / \mathrm{CoFeB}$ tunnel junctions, surprisingly showing that it happens when the spin-transfer torque is relatively small, and provides a recipe for the fabrication of magnetic tunnel junctions suitable for industrial memory applications.

pin-polarized currents flowing in conductive ferromagnets exert a pseudo-torque on the magnetization, which results from spin-momentum transfer ${ }^{1,2}$. In layered magnetic nanostructures, this spin-transfer torque (STT) effect gives rise to current-driven magnetization precession ${ }^{3}$ and even to magnetization reversal ${ }^{4,5}$. Among such nanostructures, magnetic tunnel junctions (MTJs) comprising ferromagnetic electrodes separated by an ultrathin insulating interlayer are of special interest. Indeed, MTJs are currently employed in modern hard-disk drives and represent a promising basic element for the development of an electric-write nonvolatile memory with nondestructive readout ${ }^{6}$. Importantly, they have two stable magnetic states (e.g., with parallel and antiparallel electrode magnetizations) which may display very different electrical resistance due to spin-dependent tunnelling. The phenomenon of tunnelling magnetoresistance (TMR), with TMR ratios of over $600 \%$ achieved at room temperature ${ }^{7}$, provides reliable readout of the logic state. This feature, being combined with practically unlimited endurance and very high thermal stability of information storage in MTJ-based memory cells, could make MTJs ideally suited for the next generation of non-volatile memories.

However, the current density necessary for the magnetization reversal providing the data writing is typically too high $\left(\sim 10^{6} \mathrm{~A} \mathrm{~cm}^{-2}\right)$ for industrial applications ${ }^{6,8}$. Only recently, Wang et al. discovered an easy electric-fieldassisted magnetization switching in perpendicular-anisotropy $\mathrm{Co}_{40} \mathrm{Fe}_{40} \mathrm{~B}_{20}-\mathrm{MgO}-\mathrm{Co}_{40} \mathrm{Fe}_{40} \mathrm{~B}_{20}$ junctions, which requires small current densities $\sim 10^{4} \mathrm{~A} \mathrm{~cm}^{-2}$ (ref. 9). In their experiments, a constant biasing magnetic field was applied to the junction, and reversible switching between parallel and antiparallel orientations of electrode magnetizations has been achieved by voltage pulses of the same polarity but different height. The authors attributed this unipolar switching to voltage-induced modification of perpendicular magnetic anisotropy, which can greatly reduce the coercivity. It should be emphasized that, although the electric field penetrates into metals to a distance of the order of atomic dimensions only, significant electric-field-induced changes in the surface/ interface magnetic anisotropy energy were observed experimentally and predicted theoretically for various ferromagnetic films and heterostructures ${ }^{10-15}$.

In this study, we develop a theoretical description of the voltage-driven magnetization switching in junctions having electric-field-dependent interfacial anisotropy. The theory focuses on MTJs with electrodes made of cubic ferromagnets, which are distinguished by the presence of size-induced spin reorientation transitions (SRTs) at room temperature. Special attention is given to junctions with perpendicular-to-plane magnetizations of ferromagnetic electrodes (p-MTJs), which can be stabilized by the interfacial magnetic anisotropy ${ }^{16}$ or substrateinduced lattice strains $\mathbf{u}$ (ref. 17), but qualitatively similar results are obtained for in-plane magnetized MTJs (see Supplementary Information). It is shown that the critical current density needed for the STT-driven magnetization reversal becomes small when the following three conditions are satisfied simultaneously: (i) the initial thickness-strain state of the "free" electrode is close to an SRT, (ii) the applied bias voltage enhances proximity 
to this SRT, and (iii) the junction has relatively low electrical conductance. Importantly, the application of external magnetic field is not necessary for easy voltage-driven magnetization switching, as the same role plays the magnetic coupling between two electrodes.

\section{Results}

We studied voltage-driven switching for typical MTJs having two stable magnetic states at zero bias, which correspond to parallel (P) and antiparallel (AP) electrode magnetizations. In an MTJ-based memory cell, the magnetization $\mathbf{M}$ of one electrode (termed "free" layer) changes its orientation during the writing process, whilst the magnetization $\mathbf{M}_{\text {fixed }}$ of another electrode ("fixed" layer) remains constant. For MTJs with nanoscale dimensions, we may assume that the free layer is homogeneously magnetized up to the saturation magnetization $M_{s}$ and switches through the coherent mode. Hence the magnetization dynamics in a free layer traversed by a spin-polarized current can be described by the Landau-Lifshitz-GilbertSlonczewski equation

$$
\begin{aligned}
\frac{d \mathbf{M}}{d t}= & -\gamma \mu_{0} \mathbf{M} \times \mathbf{H}_{\mathrm{eff}}-\frac{\alpha}{M_{s}} \mathbf{M} \times \frac{d \mathbf{M}}{d t} \\
& +\tau_{\mathrm{STT}} \frac{\mathbf{M} \times\left(\mathbf{M} \times \mathbf{M}_{\mathrm{fixed}}\right)}{M_{s}^{2} M_{\mathrm{fixed}}}+\tau_{\mathrm{FLT}} \frac{\mathbf{M} \times \mathbf{M}_{\mathrm{fixed}}}{M_{s} M_{\mathrm{fixed}}},
\end{aligned}
$$

where $\gamma$ is the gyromagnetic ratio, $\mu_{0}$ is the permeability of the vacuum, $\alpha$ is the dimensionless damping parameter, and $\mathbf{H}_{\mathrm{eff}}=$ $-\left(1 / \mu_{0}\right) \partial F / \partial \mathbf{M}$ is the effective magnetic field defined by the Helmholtz energy density $F$ of the free layer. The last two terms in Eq. (1) allow for the current-induced spin-transfer torque (STT) and field-like torque (FLT), respectively ${ }^{18}$. These torques depend on the voltage $V$ applied to the junction ${ }^{2,19,20}$ and should be divided by the free layer volume when substituted into Eq. (1). Importantly, in MTJs with the interfacial anisotropy sensitive to electric field in the tunnel barrier, $\mathrm{H}_{\mathrm{eff}}$ becomes a voltage-dependent quantity as well.

The analysis of Eq. (1) was restricted to free layers made of cubic ferromagnets, which have the typical (001) crystallographic orientation in the paramagnetic state. Using the expansion of the energy density $F$ in terms of direction cosines of $\mathbf{M}$ given for such layers in ref. 21 , we calculated the field $\mathbf{H}_{\text {eff. The presence of magnetic coupling }}$ between the free and fixed layers was quantified via the introduction of an effective interaction field $\mathrm{H}_{\text {int }}$ allowing for both the magnetostatic interaction ${ }^{22,23}$ and the exchange coupling. Assuming small deviations from the equilibrium magnetization orientation existing at zero applied voltage, we reduced Eq. (1) to a system of linearized equations. An analytic relation was then derived for the Jacobian matrix A of this system of equations. Using this relation, we calculated two eigenvalues of $\mathbf{A}$, which determine stability of the initial magnetic state. The analysis of these eigenvalues demonstrated that the $\mathrm{P}$ and AP states become unstable when the trace of the Jacobian, $\operatorname{tr}(\mathrm{A})$, goes to zero. Hence the critical voltage destabilizing the initial magnetic state can be found by solving the equation $\operatorname{tr}(\mathrm{A})=0$.

In the case of p-MTJs (Fig. 1), the linearized system of equations describing small deviations of $\mathbf{M}$ from the initial [001] or [00 $\overline{1}]$ direction (orientation angle $\theta=0$ or $180^{\circ}$ ) was found to be independent of the voltage-induced part of $\tau_{\mathrm{FLT}}$. Accordingly, by setting $\operatorname{tr}(\mathrm{A})$ to zero, we obtain an equation for the critical STT destabilizing the initial magnetization orientation. In the coordinate system $\left(x_{1}, x_{2}, x_{3}\right)$ with the $x_{3}$ axis orthogonal to the tunnel barrier, the critical value of $\tau_{\text {STT }}$ satisfies the relation

$$
\begin{gathered}
\tau_{\mathrm{STT}}(V)=\mp \alpha \gamma \mu_{0} M_{s}\left[H_{c}(V)+\frac{1}{2} M_{s}\left(N_{22}-N_{11}\right)+\right. \\
\left.\frac{1}{\mu_{0} M_{s}} B_{1}\left(u_{22}-u_{11}\right) \pm\left(H_{3}+H_{3}^{\mathrm{int}}\right)\right] .
\end{gathered}
$$

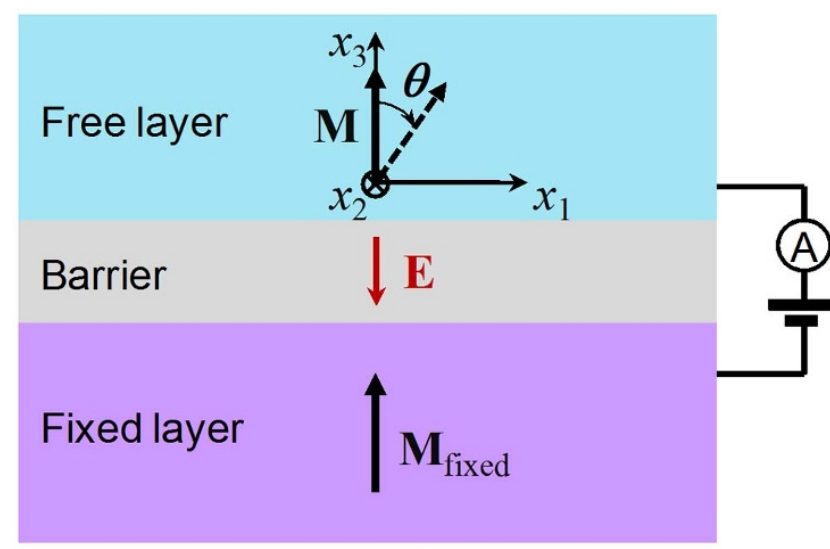

Figure 1 Schematic representation of a tunnel junction with ferromagnetic electrodes having perpendicular-to-plane magnetizations stabilized by the interfacial magnetic anisotropy or lattice strains. The magnetization $\mathbf{M}_{\mathrm{fixed}}$ of the bottom electrode has a fixed orientation assumed to be parallel to the $x_{3}$ axis, whereas the magnetization $\mathbf{M}$ of the top electrode switches between the up $\left(\theta=0^{\circ}\right)$ and down $\left(\theta=180^{\circ}\right)$ directions when the voltage applied to the junction reaches critical values.

Here and below the upper and lower signs correspond to the $\mathrm{P}$ and AP states of MTJ, respectively, $H_{3}$ is the out-of-plane component of the external magnetic field $\mathrm{H}, N_{i k}(i, k=1,2,3)$ are the demagnetizing factors of the free layer, and the critical field $H_{c}>0$ is given by the formula

$$
\begin{aligned}
H_{c}(V)= & \frac{1}{\mu_{0} M_{s}}\left[2 K_{1}+\frac{4 B_{1}^{2}}{3 c_{11}}-\frac{B_{2}^{2}}{c_{44}}+2 B_{1} u_{11}+2 B_{1} \frac{c_{12}}{c_{11}}\left(u_{11}+u_{22}\right)\right. \\
& \left.-\mu_{0} M_{s}^{2}\left(N_{33}-N_{11}\right)-\frac{2 K_{s}(V)}{t_{f}}\right] .
\end{aligned}
$$

where $K_{1}$ is the bulk magnetocrystalline anisotropy (MCA) constant of the fourth order at fixed lattice strains $\mathbf{u}$ (ref. 24), $K_{s}$ is the interfacial anisotropy constant defining the total specific energy $K_{s} M_{3}^{2} / M_{s}^{2}$ of two interfaces, $t_{f}$ is the free layer thickness, $B_{1}$ and $B_{2}$ are the magnetoelastic coefficients, and $c_{11}, c_{12}$, and $c_{44}$ are the elastic stiffnesses at constant magnetization. It should be noted that Eqs. (2)-(3) are valid even at nonzero $u_{12}$ and $N_{12}$, but the conditions $H_{1}$ $=H_{2}=H_{1}^{\text {int }}=H_{2}^{\text {int }}=0$ and $N_{13}=N_{23}=0$ must be satisfied to ensure stability of the initial magnetization orientation. In the following discussion, however, we will restrict our consideration to the usual case of zero shear strain $\left(u_{12}=0\right)$ and focus on free layers with positive MCA $\left(K_{1}>0\right)$, assuming that the magnetostatic shape anisotropy favours the [100] magnetization orientation $\left(N_{11} \leq N_{22}\right.$ $\left.\ll N_{33}, N_{12}=N_{13}=N_{23}=0\right)$ even at $u_{11} \neq u_{22}$. Importantly, under these conditions, $H_{c}$ given by Eq. (3) coincides with the theoretical coercive field of a free layer calculated from the condition $\partial^{2} F / \partial \theta^{2}=0$ at $\mathbf{H}=\mathbf{H}_{\text {int }}=0$. A similar relationship between the critical value of $\tau_{\text {STT }}$ and the coercive field can be derived for free layers with negative MCA (see Supplementary Information).

Equation (2) represents a general relation for the critical voltages $V_{\mathrm{P}}$ and $V_{\mathrm{AP}}$ of the $\mathrm{P} \rightarrow \mathrm{AP}$ and $\mathrm{AP} \rightarrow \mathrm{P}$ switching processes in the absence of thermal activation. It should be noted that, in our approximation, the field-like torque influences these voltages via a zero-bias contribution to the interaction field $\mathrm{H}_{\mathrm{int}}$ only. Based on the results of first-principles calculations ${ }^{11}$, a linear variation of the interfacial anisotropy constant $K_{s}$ with the electric field intensity $\mathrm{E}$ in the tunnel barrier can be assumed. This yields the voltage dependence of $K_{s}$ in the form $K_{s}=K_{s 0}+k_{s} V / t_{b}$, where $k_{s}=\partial K_{s} / \partial E_{3}$ is the sensitivity of interfacial anisotropy constant to the electric field, and $t_{b}$ is the barrier thickness. In this paper, the bias voltage $V$ is taken to be positive when the electric field in the barrier is directed from the free 
layer surface to that of the fixed one (Fig. 1). It should be noted that, as demonstrated by the first-principles calculations ${ }^{11,25}$, the magnetic moments of surface atoms in metallic ferromagnets also change in the presence of electric field in an adjacent dielectric. Hence the saturation magnetization $M_{s}$ in the free magnetic layer should be regarded as an electric-field-dependent quantity as well. However, for the CoFeB-MgO-CoFeB junctions with $t_{f} \sim 1.5 \mathrm{~nm}$, which are considered in this paper, the voltage-induced variations of $M_{s}$ appear to be negligible. Indeed, from the data obtained for an ultrathin $\mathrm{Fe}_{50} \mathrm{Co}_{50}$ film contacting the $\mathrm{MgO}$ layer ${ }^{25}$ it follows that the change in the free-layer magnetization $M_{s}$ is less than $1 \%$ even at the junction's breakdown field $E_{\text {break }} \approx 1.7 \mathrm{~V} \mathrm{~nm}^{-1}$ (ref. 9).

The dependence of STT on the bias voltage also can be approximated by a linear one (constant torkance) ${ }^{2}$. Accordingly, we can represent $\tau_{\mathrm{STT}}$ as $\tau_{\mathrm{STT}}=\gamma(\hbar / e) G_{\mathrm{STT}} V / t_{f}$, where $e$ is the electron charge, $\hbar$ is the Planck constant, and $G_{\mathrm{STT}}$ is the normalized torkance having the dimension of conductance per unit area. The theoretical studies demonstrate that $G_{\text {STT }}$ should strongly depend on the height $U_{b}$ and width $t_{b}$ of potential barrier, electron effective mass $m_{b}$ in the tunnel barrier (which may differ markedly from the free electron mass $m_{e}$ ), and on physical properties of electrodes ${ }^{2,26}$. Since both STT and junction's electric conductance $G$ are proportional to the exponential factor $\exp \left(-2 t_{b} \sqrt{2 m_{b} U_{b}} / \hbar\right)$ (ref. 26), a strong correlation between the torkance $G_{\text {STT }} \sim \exp \left(-2 t_{b} \sqrt{2 m_{b} U_{b}} / \hbar\right)$ and the conductance $G$ is expected. In particular, for elastic tunneling in symmetric MTJs, the theory gives $G_{\text {STT }}=(1 / 2) G_{\mathrm{P}} P /\left(1+P^{2}\right)$, where $P$ is the spin polarization, and $G_{\mathrm{P}}$ is the MTJ conductance per unit area in the $\mathrm{P}$ state $^{2}$.

In the linear approximation, the critical voltages defined by Eq. (2) become

$$
\begin{aligned}
V_{\mathrm{P}, \mathrm{AP}} \approx & \mp \frac{\alpha t_{f}}{(\hbar / e) G_{\mathrm{STT}} \mp 2 \alpha k_{s} / t_{b}} \mu_{0} M_{s} \\
& {\left[H_{c 0}+\frac{1}{2} M_{s}\left(N_{22}-N_{11}\right)+\frac{B_{1}}{\mu_{0} M_{s}}\left(u_{22}-u_{11}\right) \pm\left(H_{3}+H_{3}^{\mathrm{int}}\right)\right], }
\end{aligned}
$$

where $H_{c 0}=H_{c}(V=0)$. Therefore, the critical current densities for the $\mathrm{P} \rightarrow \mathrm{AP}$ and $\mathrm{AP} \rightarrow \mathrm{P}$ switching processes can be found as $J_{\mathrm{P}}=$ $G_{\mathrm{P}}\left(V_{\mathrm{P}}\right) V_{\mathrm{P}}$ and $J_{\mathrm{AP}}=G_{\mathrm{AP}}\left(V_{\mathrm{AP}}\right) V_{\mathrm{AP}}$, where $G_{\mathrm{AP}}$ is the MTJ conductance per unit area in the AP state. Equation (4) shows that the voltage-dependent interfacial anisotropy facilitates one of the switching processes $(\mathrm{P} \rightarrow \mathrm{AP}$ or $\mathrm{AP} \rightarrow \mathrm{P})$ and hinders the other. Since the magnetization of the fixed layer usually can be reversed by sufficiently strong magnetic field, it is possible to magnetize an MTJ in such a way that the current-induced switching hindered by the interlayer magnetic coupling will be facilitated by the interfacial anisotropy.

According to the available experimental data ${ }^{9,15}$, in the case of $\mathrm{CoFeB}-\mathrm{MgO}-\mathrm{CoFeB}$ junctions the sensitivity $k_{s}$ should be negative in our free-energy formulation, where the out-of-plane magnetization orientation is favoured by negative $K_{s}$. Hence the electric-fielddependent interfacial anisotropy facilitates $\mathrm{P} \rightarrow \mathrm{AP}$ switching and hinders $\mathrm{AP} \rightarrow \mathrm{P}$ one in these MTJs. Using typical values of $k_{s} \approx$ $-50 \mu \mathrm{J} \mathrm{m}^{-2}\left(\mathrm{~V} \mathrm{~nm}^{-1}\right)^{-1}, \alpha \sim 0.01$, and $t_{b} \sim 1 \mathrm{~nm}$, we obtain $\left|2 \alpha k_{s}\right|$ $t_{b} \mid \sim 10^{-6} \mathrm{C} / \mathrm{m}^{2}$. At the same time, the measured voltage dependence of STT (ref. 19) demonstrates that, for the $\mathrm{Co}_{60} \mathrm{Fe}_{20} \mathrm{~B}_{20}-\mathrm{MgO}$ $\mathrm{Co}_{60} \mathrm{Fe}_{20} \mathrm{~B}_{20}$ junction with $t_{b}=1 \mathrm{~nm}$, the normalized torkance $G_{\text {STT }}$ is about $6 \times 10^{10} \Omega^{-1} \mathrm{~m}^{-2}$ at small voltages. Therefore, $(\hbar /$ e) $G_{\mathrm{STT}} \approx 4 \times 10^{-5} \mathrm{C} / \mathrm{m}^{2}$ so that the first term in the denominator of Eq. (4) appears to be much larger than the second one in this particular case. However, the conductance of this MTJ is very high $\left(G_{\mathrm{P}} \sim 5 \times 10^{11} \Omega^{-1} \mathrm{~m}^{-2}\right)^{19}$, which indicates that the STT is much higher here than in junctions with small conductances. For instance, the $\mathrm{Co}_{40} \mathrm{Fe}_{40} \mathrm{~B}_{20}-\mathrm{MgO}-\mathrm{Co}_{40} \mathrm{Fe}_{40} \mathrm{~B}_{20}$ junctions with $t_{b}=1.2 \mathrm{~nm}$ have $G_{\mathrm{P}} \sim 10^{8} \Omega^{-1} \mathrm{~m}^{-2}$ (ref. 9) so that the second term is expected to be much larger than the first one, which implies strong effect of the electric-field-dependent interfacial anisotropy on critical voltages and currents.

Importantly, the coercive field $H_{c 0}$ involved in Eq. (4) decreases drastically in the vicinity of size-induced or strain-driven SRT. At $K_{1}$ $>0$, such SRT has the form of an abrupt transition between perpendicular-to-plane and in-plane magnetization states ${ }^{21,24}$, and the relation $\mu_{0} M_{s} H_{c 0}=2 K_{1}+\left(B_{1}^{2} / c_{11}\right)-\left(B_{2}^{2} / c_{44}\right)-2 K_{s 0}\left(t^{*}-t_{f}\right) /\left(t^{*} t_{f}\right)$ can be derived for the coercive field of free layer at $\mathrm{H}=\mathrm{H}_{\text {int }}=0$, where $t^{*}$ is the critical thickness at $V=0$ given by Eq. (S4) (see Supplementary Information). We see that $H_{\mathrm{c} 0}$ reduces as $t_{f}$ tends to $t^{*}$ but remains finite at this first-order transition. Nevertheless, the coercive field $H_{\mathrm{c} 0}$ and, therefore, the critical voltages and currents can be strongly reduced by fabricating MTJs with an optimum free layer thickness close to $t^{*}$.

To demonstrate this effect, we calculated the critical voltage and current for the $\mathrm{P} \rightarrow \mathrm{AP}$ switching in a representative $\mathrm{CoFeB} / \mathrm{MgO} /$ $\mathrm{CoFeB}$ junction. The torkance $G_{\mathrm{STT}}$ was evaluated as a function of the junction's conductance $G_{\mathrm{P}}$ under the assumption of elastic tunneling $^{2}$ for the spin polarization $P=0.577$ corresponding to $\mathrm{TMR}=$ $100 \%$. The relationship between $G_{\mathrm{P}}$ and the barrier thickness $t_{b}$ was described by the formula $G_{\mathrm{P}}=G_{\mathrm{P} 0} \exp \left(-2 t_{b} \sqrt{2 m_{b} U_{b}} / \hbar\right)$ with the height $U_{b}=1.2 \mathrm{eV}$ and effective mass $m_{b}=0.4 m_{e}$ characteristic of the $\mathrm{MgO}$ barrier $^{26}$ and $G_{\mathrm{P} 0}$ providing the conductance $G_{\mathrm{P}}=7.96 \times$ $10^{7} \Omega^{-1} \mathrm{~m}^{-2}$ measured at $t_{b}=1.2 \mathrm{~nm}$ (ref. 9). Substituting $G_{\mathrm{STT}}\left(G_{\mathrm{P}}\right)$ and $t_{b}\left(G_{\mathrm{P}}\right)$ into Eq. (4), we found the critical voltage $V_{\mathrm{P}}$ and current density $J_{\mathrm{P}}$ as a function of the junction's conductance $G_{\mathrm{P}}$. Figure 2 shows that, irrespective of this conductance, both $V_{\mathrm{P}}$ and $J_{\mathrm{P}}$ decrease significantly as the free layer thickness $t_{f}$ approaches the critical value $t^{*} \cong 1.67 \mathrm{~nm}$. This decrease results from a thickness-induced reduction of the coercive field $H_{c 0}\left(t_{f}\right)=H_{c 0}\left(t^{*}\right)-2\left(K_{s 0} / \mu_{0} M_{s}\right)$ $\left(t^{*}-t_{f}\right) /\left(t^{*} t_{f}\right)$, which leads to a term proportional to $K_{s 0}\left(t^{*}-t_{f}\right)$ $/ t^{*}$ in Eq. (4). It should be noted that, since $t^{*} \sim K_{s 0}$, both the minimum critical voltage $V_{\mathrm{P}}\left(t^{*}\right)$ and the slope $\partial V_{\mathrm{P}} / \partial t_{f}$ of its thickness dependence are independent of the interfacial anisotropy constant $K_{s 0}$. Indeed, the same feature is characteristic of the critical current density $J_{\mathrm{P}}$.

On the other hand, the reduction of conductance is accompanied by a drastic decrease in the current density $J_{\mathrm{P}}$ and increase in the voltage $V_{\mathrm{P}}$ at all studied thicknesses $t_{f}$. Remarkably, very low critical current densities $J_{\mathrm{P}} \leq 10^{4} \mathrm{~A} \mathrm{~cm}^{-2}$ are predicted for junctions with $G_{\mathrm{P}}$ $\leq 10^{8} \Omega^{-1} \mathrm{~m}^{-2}$. However, such easy current-induced switching can be realized only when the free layer thickness $t_{f}$ is sufficiently close to $t^{*}$ so that the voltage $V_{\mathrm{P}}$ does not exceed the junction's breakdown voltage $V_{\text {break. }}$.

It should be emphasized that the bias voltage $V$ applied to a ferromagnetic layer with electric-field-dependent interfacial anisotropy can induce an SRT as well ${ }^{11}$. In the case of negligible interlayer coupling and $K_{1}>0$, the calculation gives the following relation for the voltage $V^{*}$ at which the perpendicular-to-plane and in-plane magnetization states acquire the same energy:

$$
\begin{aligned}
V^{*}= & \frac{t_{f} t_{b}}{k_{s}}\left[-\frac{K_{s 0}}{t_{f}}+\frac{B_{1}^{2}}{6 c_{11}}+B_{1} u_{11}+B_{1} \frac{c_{12}}{c_{11}}\left(u_{11}+u_{22}\right)\right. \\
& \left.-\frac{1}{2} \mu_{0} M_{s}^{2}\left(N_{33}-N_{11}\right)\right] .
\end{aligned}
$$

Since at $V=V^{*}$ the energy minima corresponding to these states are separated by a potential barrier, the $90^{\circ}$ magnetization switching actually occurs at a higher bias voltage $\left(|V|>\left|V^{*}\right|\right)$, which makes the initial state unstable (by transforming an energy minimum into a maximum) or reduces the barrier height to a level surmountable by thermal fluctuations. Moreover, expressing $V^{*}$ through the critical thickness $t^{*}$, we find that $V^{*}=-\left(K_{s 0} t_{b} / k_{s}\right)\left(t^{*}-t_{f}\right) / t^{*}$. Therefore, the switching voltage is expected to be smaller than the MTJ breakdown voltage only at thicknesses $t_{f}$ close to $t^{*}$. In the case of CoFeB$\mathrm{MgO}-\mathrm{CoFeB}$ junctions, for instance, $V_{\text {break }} \approx 2 \mathrm{~V}$ (ref. 9) and $K_{s 0} \approx$ 

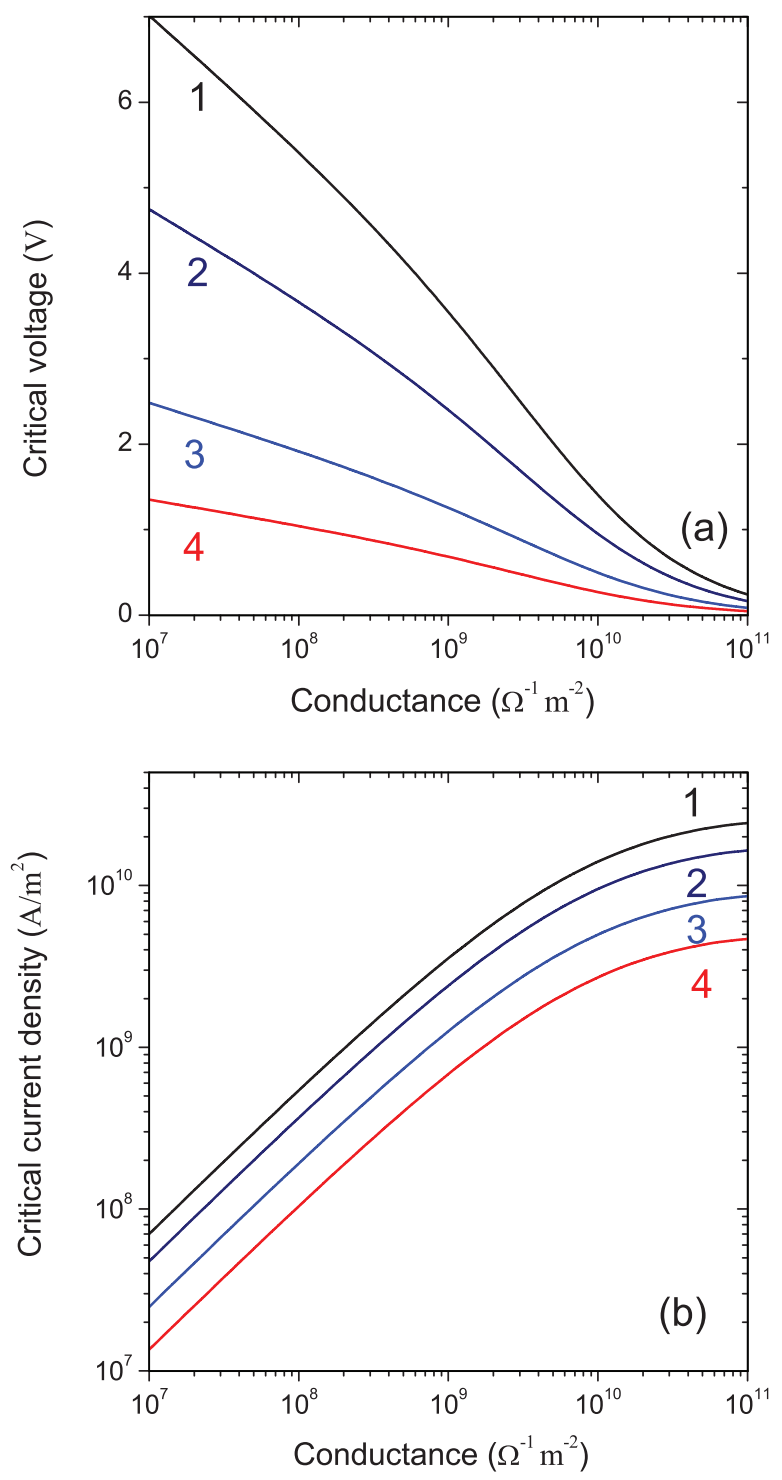

Figure $2 \mid$ Theoretical results for spin-transfer-driven magnetization switching in $\mathrm{CoFeB} / \mathrm{MgO} / \mathrm{CoFeB}$ junctions. (a) Critical voltage and (b) critical current density for the $\mathrm{P} \rightarrow \mathrm{AP}$ switching calculated as a function of the junction's conductance per unit area in the P state. Curves 1, 2, 3, and 4 correspond to the free layer thicknesses $t_{f}=1.4,1.5,1.6$, and $1.65 \mathrm{~nm}$, respectively. The following parameters were employed in the numerical calculations: $H_{3}^{\text {int }}=50$ Oe, $N_{11}=N_{22}=0.01, u_{11}=u_{22}=0, \alpha=0.01, K_{s 0}$ $=-1.3 \times 10^{-3} \mathrm{~J} \mathrm{~m}^{-2}$ (ref. 16), $k_{s}=-50 \mu \mathrm{J} \mathrm{m}^{-2}\left(\mathrm{~V} \mathrm{~nm}^{-1}\right)^{-1}, M_{s}=1.13 \times$ $10^{6} \mathrm{~A} \mathrm{~m}^{-1}$ (ref. 9), $K_{1}=1.3 \times 10^{4} \mathrm{~J} \mathrm{~m}^{-3}, B_{1}=-29.4 \times 10^{6} \mathrm{~J} \mathrm{~m}^{-3}, B_{2}=-3$ $\times 10^{6} \mathrm{~J} \mathrm{~m}^{-3}, c_{11}=2.8 \times 10^{11} \mathrm{~N} \mathrm{~m}^{-2}, c_{12}=1.4 \times 10^{11} \mathrm{~N} \mathrm{~m}^{-2}$, and $c_{44}=1 \times$ $10^{11} \mathrm{~N} \mathrm{~m}^{-2}$ (ref. 21).

$-1.3 \times 10^{-3} \mathrm{~J} \mathrm{~m}^{-2}$ (ref. 16) so that the proximity $\left(t^{*}-t_{f}\right) / t^{*}$ to the SRT must be less than $10 \%$.

To reveal peculiarities of the magnetization switching occurring in the presence of interlayer coupling or external magnetic field, we analyze the voltage-induced evolution of the free layer energy density $\Delta F$ as a function of the angle $\theta$ between the magnetization $M$ and the $x_{3}$ axis. Figure 3 shows representative energy profiles $\Delta F(\theta)$ calculated for a $\mathrm{CoFeB}-\mathrm{MgO}-\mathrm{CoFeB}$ junction with the interlayer coupling characterized by the interaction field $H_{3}^{\text {int }}=50$ Oe. It can be seen that, at $V=0$ and at negative voltages not exceeding the threshold $V_{\text {th }} \cong-1.02 \mathrm{~V}$, the energy minima exist only at $\theta=0^{\circ}$ and $\theta=180^{\circ}$, which correspond to the perpendicular-to-plane magnetization orientations parallel and antiparallel to the magnetization of the fixed
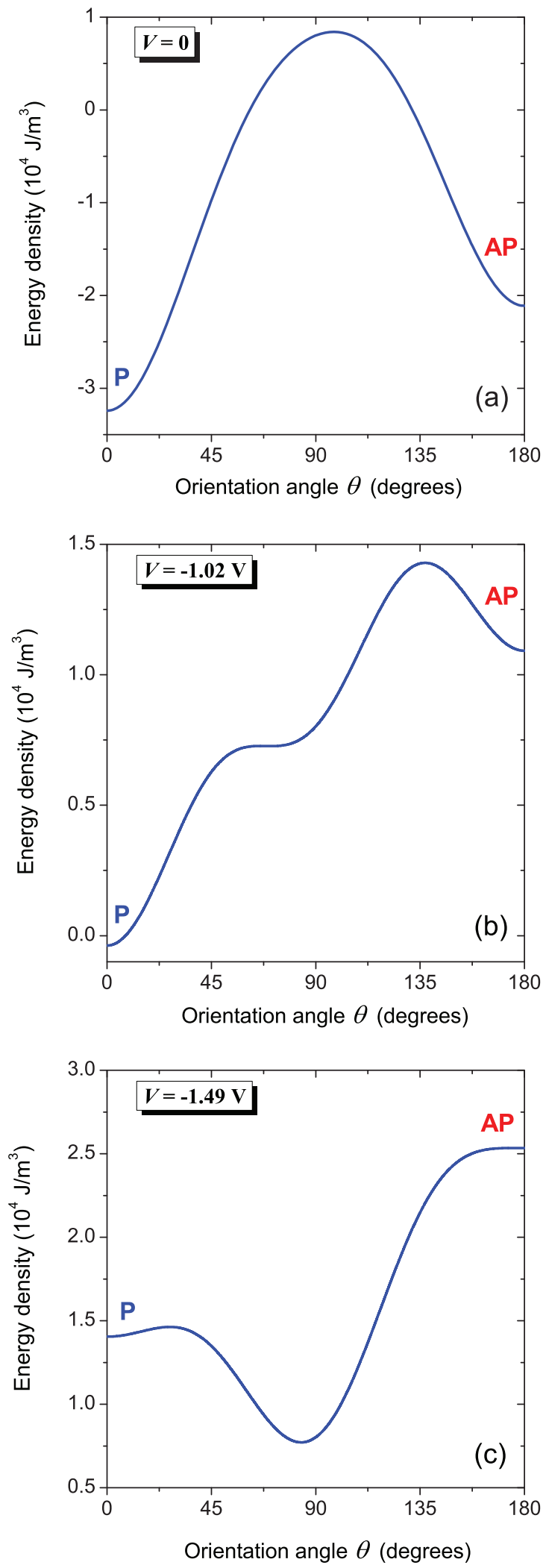

Figure 3 Effect of bias voltage on the energetics of a free magnetic layer in a $\mathrm{CoFeB}-\mathrm{MgO}$ junction with nonzero interlayer magnetic coupling. The free layer energy density $\Delta F$ is plotted as a function of the magnetization orientation angle $\theta$ shown in Fig. 1. Panel (a) corresponds to the initial state of this junction $(V=0)$, whilst panels (b) and (c) demonstrate the energy profiles at bias voltages of $-1.02 \mathrm{~V}$ and $-1.49 \mathrm{~V}$, respectively. The calculations were performed for a junction with $H_{3}^{\text {int }}=50 \mathrm{Oe}, t_{f}=1.6 \mathrm{~nm}$, and $t_{b}=1 \mathrm{~nm}$ using the set of parameters listed in the caption of Fig. 2. 
layer, respectively. At higher negative voltages, however, an intermediate minimum corresponding to almost in-plane magnetization direction appears at $70^{\circ}<\theta<90^{\circ}$. Furthermore, at $V=V_{c} \cong$ $-1.49 \mathrm{~V}$, the energy minimum at $\theta=180^{\circ}$, which is related to the AP state disadvantaged by the interlayer coupling, transforms into a maximum. Therefore, the voltage $V_{c}$ can induce a switching of the free layer magnetization in an MTJ having initially the AP state. In this case, the free layer magnetization $\mathrm{M}$ will rotate by about $96^{\circ}$ and acquire almost in-plane orientation. Remarkably, the reduction of voltage back to $V_{\text {th }}$ will give rise to further magnetization rotation towards $\theta=0^{\circ}$ because the intermediate minimum disappears at this voltage. Thus, a voltage pulse of sufficient height $\left(|V|>\left|V_{c}\right|\right)$ can induce the $\mathrm{AP} \rightarrow \mathrm{P}$ switching facilitated by interlayer coupling. It should be noted that the influence of STT on the AP $\rightarrow P$ switching in a CoFeB-MgO-CoFeB junction with $H_{3}^{\text {int }}=50$ Oe and a low conductance $G_{\mathrm{P}}<3 \times 10^{8} \Omega^{-1} \mathrm{~m}^{-2}$ can be neglected because the ratio $\tau_{\mathrm{STT}} /\left(\gamma \mu_{0} M_{s} H_{3}^{\text {int }}\right)$ is less than $1 \%$ even at the bias voltage $V=-2 \mathrm{~V}$ close to the breakdown voltage. A similar voltage-assisted $180^{\circ} \mathrm{mag}$ netization rotation was observed in the $\mathrm{Co}_{40} \mathrm{Fe}_{40} \mathrm{~B}_{20}-\mathrm{MgO}$ $\mathrm{Co}_{40} \mathrm{Fe}_{40} \mathrm{~B}_{20}$ junctions subjected to an external magnetic field ${ }^{9}$.

At the same time, the appearance of an intermediate energy minimum between $\theta=0^{\circ}$ and $\theta=180^{\circ}$ might interrupt the $\mathrm{P} \rightarrow \mathrm{AP}$ switching initiated by the spin-polarized current. Indeed, the STT and FLT torques destabilizing the initial P state may be insufficient for the escape from this energy minimum. Then, at the critical voltage $V_{\mathrm{P}}$ given by Eq. (4), the free layer magnetization $\mathbf{M}$ will acquire almost in-plane orientation instead of being rotated by $180^{\circ}$. Moreover, owing to the interlayer magnetic coupling, $\mathbf{M}$ will rotate back to the initial direction after completion of the voltage pulse. In this situation, which is beyond the scope of the present paper, the actual voltage needed for the $\mathrm{P} \rightarrow \mathrm{AP}$ switching may be higher than $V_{\mathrm{P}}$. Hence Eq. (4) can be safely used only when it gives the critical voltage not exceeding significantly the voltage $V_{\text {th }}(\approx-1.02 \mathrm{~V}$ for the considered MTJ). At $\left|\mathrm{H}_{\text {int }}\right| \ll M_{s}, V_{\text {th }}$ can be approximated by the voltage $V^{*}$ defined by Eq. (5).

Remarkably, our theory explains easy magnetization switching in the $\mathrm{Co}_{40} \mathrm{Fe}_{40} \mathrm{~B}_{20}-\mathrm{MgO}-\mathrm{Co}_{40} \mathrm{Fe}_{40} \mathrm{~B}_{20}$ junctions, which was recently discovered by Wang et $\mathrm{al}^{9}$. In this work, p-MTJs with the free layer thickness $t_{f}=1.6 \mathrm{~nm}$ and the barrier thickness $t_{b}=1.2 \mathrm{~nm}$ were subjected to voltage pulses in the presence of external magnetic field $\mathrm{H}_{3}=55 \mathrm{Oe}$. It was found that the sequence of unipolar pulses with the duration $\delta_{p}=200 \mathrm{~ms}$ and magnitude alternating between $-0.9 \mathrm{~V}$ and $-1.5 \mathrm{~V}$ reversibly switches this MTJ between $\mathrm{P}$ and AP states. The $\mathrm{P} \rightarrow \mathrm{AP}$ reversal was attributed to an STT created by the spin-polarized current, whilst the $\mathrm{AP} \rightarrow \mathrm{P}$ switching was explained by a voltage-induced reduction of coercive field ${ }^{9}$. At the same time, the substitution of $K_{s 0}=-1.3 \times 10^{-3} \mathrm{~J} \mathrm{~m}^{-2}$ (ref. 16), $M_{s}$ $=1.13 \times 10^{6} \mathrm{~A} \mathrm{~m}^{-1}$ (ref. 9), $u_{m}=0, N_{11}=0.008$, and $N_{33}=0.984$ (theoretical values for the free layer with diameter $D=400 \mathrm{~nm}$ ) into Eq. (S4) shows that, for the discussed MTJ, the critical thickness $t^{*}$ in the absence of magnetic field and interlayer coupling is equal to $t^{*} \approx$ $1.66 \mathrm{~nm}$. Accordingly, the free layer with $t_{f}=1.6 \mathrm{~nm}$ is very close to a thickness-driven SRT already at $V=0$. By analyzing the influence of bias voltage on the energy profile $\Delta F(\theta)$ at $H_{3}=55$ Oe and the measured sensitivity $k_{s}=-50 \mu \mathrm{J} \mathrm{m}^{-2}\left(\mathrm{~V} \mathrm{~nm}^{-1}\right)^{-1}$ (ref. 9) we further find that the threshold voltage $V_{\text {th }}$ is about $-1.08 \mathrm{~V}$, whilst the critical voltage $V_{c}$ at which the AP state becomes unstable at temperature $T=0 \mathrm{~K}$ equals $V_{c} \cong-1.59 \mathrm{~V}$. Hence the $\mathrm{AP} \rightarrow \mathrm{P}$ switching observed at $V=-1.5 \mathrm{~V}$ may be attributed to the two-stage magnetization rotation via an intermediate orientation which becomes unstable when the bias voltage reduces below $V_{\text {th }} \cong-1.08 \mathrm{~V}$ during the voltage downswing. The difference between the observed switching voltage and the theoretical critical voltage $V_{c}(T=0)$ may be explained by the influence of thermal fluctuations. Indeed, the Néel-Brown formula ${ }^{27}$ for the switching probability $\Pi(T)$ shows that $V_{c}(T)$ can be estimated using the equality $U_{b}(V)=k_{B} T \ln \left(\delta_{p} f_{0} / \xi\right)$, where $U_{b}$ is the height of the barrier hindering the escape of magnetization from the minimum at $\theta=180^{\circ}, f_{0} \sim 10^{\circ} \mathrm{Hz}$ is the attempt frequency, and the factor $\xi \cong 5$ ensures $\Pi \cong 1$. By studying the voltage-induced evolution of the energy profile $\Delta F(\theta)$ we found that $V_{c} \cong-1.4 \mathrm{~V}$ at $T=300 \mathrm{~K}$ and $\delta_{p}=200 \mathrm{~ms}$, which agrees well with the experiment.

The critical current needed for the STT-driven magnetization switching reduces significantly in the presence of thermal activation as well ${ }^{6}$. From the general theory of thermally assisted magnetization reversal ${ }^{28}$ it follows that the critical STT strength at a finite temperature is given by the formula $\tau_{\mathrm{STT}}(T)=\tau_{\mathrm{STT}}(T=0)\left[1-k_{B} T \ln \left(\delta_{p} f_{0} /\right.\right.$ $\left.\xi) / U_{b}\right]$, where $\tau_{\mathrm{STT}}(T=0)$ is defined by Eq. (2). Accordingly, the critical voltage $V_{\mathrm{P}}(T)$ of the $\mathrm{P} \rightarrow \mathrm{AP}$ switching calculated in the linear approximation differs from $V_{\mathrm{P}}(T=0)$ by the factor $1-k_{B} T \ln \left(\delta_{p} f_{0} /\right.$ $\xi) / U_{b}$. Since the discussed MTJ has the conductance $G_{\mathrm{P}} \approx 1.3 \times$ $10^{8} \Omega^{-1} \mathrm{~m}^{-2}$ and the TMR $=100 \%$ corresponding to spin polarization $P \approx 0.577$ (ref. 9), the normalized torkance $G_{\text {STT }}$ calculated for elastic tunneling ${ }^{20}$ is about $2.9 \times 10^{7} \Omega^{-1} \mathrm{~m}^{-2}$. Hence, using the measured barrier height $U_{b}=42 k_{B} T$ (ref. 9) and damping constant $\alpha=0.013$ (ref. 16), we obtain $V_{\mathrm{P}} \approx-1.05 \mathrm{~V}$ and $J_{\mathrm{P}} \approx-1.4 \times 10^{4} \mathrm{~A}$ $\mathrm{cm}^{-2}$. Remarkably, the predicted ultralow critical current density is very close to the measured value $J_{\mathrm{P}}=-1.2 \times 10^{4} \mathrm{~A} \mathrm{~cm}^{-2}$ (ref. 9), which is two orders of magnitude smaller than usual densities $\sim 10^{6} \mathrm{~A} \mathrm{~cm}^{-2}$ (ref. 16). It should be noted that the critical current density calculated without the account of voltage-controlled interfacial anisotropy $\left(k_{s}=0\right)$ is about $-8 \times 10^{5} \mathrm{~A} \mathrm{~cm}^{-2}$.

\section{Discussion}

In this work, we have shown that the electric-field-dependent interfacial magnetic anisotropy has a strong effect on the STT-driven magnetization reversal in MTJs with a low conductance. Depending on the sign of its sensitivity $k_{s}$ to electric field, the varying interfacial anisotropy facilitates either the $\mathrm{P} \rightarrow \mathrm{AP}$ or $\mathrm{AP} \rightarrow \mathrm{P}$ switching, reducing the critical current density down to ultralow values $\leq 10^{4} \mathrm{~A}$ $\mathrm{cm}^{-2}$. Surprisingly, this easy magnetization reversal occurs when the spin-transfer torque is rather small. Although the reverse process may be strongly hindered and even prohibited by the change of interfacial anisotropy, it can be realized via the voltage-induced two-stage magnetization rotation facilitated by the interlayer magnetic coupling. Such switching, however, is an exclusive feature of tunnel junctions distinguished by the proximity of free layer to the size-induced SRT. Thus, the theory provides a recipe for the fabrication of MTJs suitable for commercial electric-write nonvolatile magnetic random access memories.

1. Slonczewski, J. C. Conductance and exchange coupling of two ferromagnets separated by a tunneling barrier. Phys. Rev. B 39, 6995-7002 (1989).

2. Slonczewski, J. C. \& Sun, J. Z. Theory of voltage-driven current and torque in magnetic tunnel junctions. J. Magn. Magn. Mater. 310, 169-175 (2007).

3. Kiselev, S. I. et al. Microwave oscillations of a nanomagnet driven by a spinpolarized current. Nature 425, 380-383 (2003).

4. Myers, E. B., Ralph, D. C., Katine, J. A., Louie, R. N. \& Buhrman, R. A. Current induced switching of domains in magnetic multilayer devices. Science 285, 867-870 (1999).

5. Huai, Y., Albert, F., Nguyen, P., Pakala, M. \& Valet, T. Observation of spintransfer switching in deep submicron-sized and low-resistance magnetic tunnel junctions. Appl. Phys. Lett. 84, 3118-3120 (2004).

6. Ikeda, S. et al. Magnetic tunnel junctions for spintronic memories and beyond. IEEE Trans. Electron Devices 54, 991-1002 (2007).

7. Ikeda, S. et al. Tunnel magnetoresistance of $604 \%$ at $300 \mathrm{~K}$ by suppression of $\mathrm{Ta}$ diffusion in $\mathrm{CoFeB} / \mathrm{MgO} / \mathrm{CoFeB}$ pseudo-spin-valves annealed at high temperature. Appl. Phys. Lett. 93, 082508 (2008).

8. Ralph, D. C. et al. Spin-transfer torque in nanoscale magnetic devices. Phil. Trans. R. Soc. A 369, 3617-3630 (2011).

9. Wang, W.-G., Li, M., Hageman, S. \& Chien, C. L. Electric-field-assisted switching in magnetic tunnel junctions. Nature Mater. 11, 64-68 (2011).

10. Weisheit, M. et al. Electric field-induced modification of magnetism in thin-film ferromagnets. Science 315, 349-351 (2007).

11. Duan, C.-G. et al. Surface magnetoelectric effect in ferromagnetic metal films Phys. Rev. Lett. 101, 137201 (2008). 
12. Maruyama, T. et al. Large voltage-induced magnetic anisotropy change in a few atomic layers of iron. Nature Nanotech. 4, 158-161 (2009).

13. Nakamura, K. et al. Giant modification of the magnetocrystalline anisotropy in transition-metal monolayers by an external electric field. Phys. Rev. Lett. 102, 187201 (2009).

14. Niranjan, M. K., Duan, C.-G., Jaswal, S. S. \& Tsymbal, E. Y. Electric field effect on magnetization at the Fe-MgO(001) interface. Appl. Phys. Lett. 96, 222504 (2010).

15. Kanai, S. et al. Electric field-induced magnetization reversal in a perpendicularanisotropy CoFeB-MgO magnetic tunnel junction. Appl. Phys. Lett. 101, 122403 (2012).

16. Ikeda, S. et al. A perpendicular-anisotropy $\mathrm{CoFeB}-\mathrm{MgO}$ magnetic tunnel junction. Nature Mater. 9, 721-724 (2010).

17. Schulz, B. \& Baberschke, K. Crossover from in-plane to perpendicular magnetization in ultrathin Ni/Cu(001) films. Phys. Rev. B 50, 13467-13471 (1994).

18. Brataas, A., Kent, A. D. \& Ohno, H. Current-induced torques in magnetic materials. Nature Mater. 11, 372-381 (2012).

19. Kubota, H. et al. Quantitative measurement of voltage dependence of spintransfer torque in $\mathrm{MgO}$-based magnetic tunnel junctions. Nature Phys. 4, 37-41 (2008).

20. Sankey, J. C. et al. Measurement of the spin-transfer-torque vector in magnetic tunnel junctions. Nature Phys. 4, 67-71 (2008).

21. Pertsev, N. A. \& Kohlstedt, H. Magnetoresistive memory with ultralow critical current for magnetization switching. Adv. Funct. Mater. 22, 4696-4703 (2012).

22. Moon, K.-S., Fontana, R. E., Jr. \& Parkin, S. S. P. Exchange-biased magnetic tunnel junctions: Dependence of offset field on junction width. Appl. Phys. Lett. 74, 3690-3692 (1999).

23. Schrag, B. D. et al. Néel “orange-peel” coupling in magnetic tunneling junction devices. Appl. Phys. Lett. 77, 2373-2375 (2000).

24. Pertsev, N. A. Giant magnetoelectric effect via strain-induced spin-reorientation transitions in ferromagnetic films. Phys. Rev. B 78, 212102 (2008)
25. He, K. H., Chen, J. S. \& Feng, Y. P. First principles study of the electric field effect on magnetization and magnetic anisotropy of $\mathrm{FeCo} / \mathrm{MgO}(001)$ thin film. Appl. Phys. Lett. 99, 072503 (2011).

26. Xiao, J., Bauer, G. E. W. \& Brataas, A. Spin-transfer torque in magnetic tunnel junctions: Scattering theory. Phys. Rev. B 77, 224419 (2008).

27. Brown, W. F., Jr. Thermal fluctuations of a single-domain particle. Phys. Rev. 130, 1677-1686 (1963).

28. Li, Z. \& Zhang, S. Thermally assisted magnetization reversal in the presence of a spin-transfer torque. Phys. Rev. B 69, 134416 (2004).

\section{Acknowledgments}

The financial support provided via the ERA.Net RUS project NANO-C (STProjects-133) is gratefully acknowledged. This work was also supported by the grant No. 14.B25.31.0025 of the Government of the Russian Federation.

\section{Additional information}

Supplementary information accompanies this paper at http://www.nature.com/ scientificreports

Competing financial interests: The author declares no competing financial interests.

How to cite this article: Pertsev, N.A. Origin of easy magnetization switching in magnetic tunnel junctions with voltage-controlled interfacial anisotropy. Sci. Rep. 3, 2757;

DOI:10.1038/srep02757 (2013).

(c) (i) $(-)$ This work is licensed under a Creative Commons Attribution-

cc) visit http://creativecommons.org/licenses/by-nc-nd/3.0 